\title{
A WiFi and PLC Based Smart Home Control System
}

\author{
Huang Risheng \\ School of Electronic and Information Engineering, Heyuan Polytechnic, Heyuan 517000, China \\ Email: huangrisheng_1982@163.com
}

\begin{abstract}
Keywords: Smart home; Power Line Communication (PLC); Wireless local area network; Network mode

Abstract. Among the existing network modes in smart home, wired network is not easy to construct and the workload is heavy, and wireless network has shortcomings such as high power and high radiation. In order to solve this problem, this paper proposes a new network scheme for smart home based on WiFi and low voltage power carrier communication. This paper describes the design of the whole household system, the combination of $\mathrm{WiFi}$ and power line communication, the design of electric power carrier terminal module and control terminal software and so on. Actual tests prove the system realizes the complementary advantages of the two kinds of communication technology. The system can monitor the state of the electronic equipment and control them in real time reliably. It will have a good market prospect.
\end{abstract}

\section{Introduction}

With the continuous development of the Internet of things (IoT) [1] and a variety of sensor technology, wireless network communication technology, people's requirements on all aspects of daily life gradually increased, and the "Smart home" concept has been popular in recent years.

Wiki encyclopedia gives the "Smart home" [2] specific concepts: it is a platform with residential, with building automation, network communication, information appliances and equipment, system, structure, service and management as one of the efficient, comfortable, safe, convenient and environmentally living environment. After entering in the 21 st Century, with the continuous development of technology, its development also tends to be diversified. At present, many domestic and foreign enterprises and universities have carried out in-depth research and testing in the field of smart home. Among them, paper [3] puts forward a intelligent home control system design based on GSM short message, it is simple, practical and cheap, but its function is single, and the user can only operate through a fixed message format, which is not a good experience; paper [4] proposed a design scheme using 433 wireless module to build home internal network, it has the advantages of low cost, low power consumption and small size package, but the limitation of use external GSM network has not been changed; paper [5-6] presents the application of PLC in the internal network of smart home, and compared the PLC with respect to the advantages of Ethernet, telephone line and wireless networks, but only limited to the internal network, not the whole structure of smart home to make elaborate; paper [7] proposed a design scheme of smart home system based on ZigBee and PLC, to a certain extent, this method achieves the advantages of the 2 technologies and it is a good feasible scheme, however, due to the popularity of ZigBee technology and the WiFi co-channel interference problems [8], there will be lots of defects while using in house. As all kinds of limitations in smart home networking technology, we propose a smart home network technology based on WiFi and PLC, as we know, $\mathrm{WiFi}$ is widely popular and has the unique advantage of connecting to the Internet, and PLC has the advantage of no need to re-wiring, little transformation of the old home, combining the two and make use the advantages of them, provides a feasible scheme for the construction of efficient, convenient and smart home system.

\section{The Architecture of Smart Home Network}

WiFi Technology and Wireless Module WG1300. WiFi, also known as wireless compatibility authentication, is a device that does not have to be connected by wires [9]. At present, it has been widely used in homes, businesses and other public places, such as, its main principle is using 
electromagnetic waves to achieve different devices in a wireless network data transmission and sharing, common transmission device including the antenna and the router, the receiving device includes wireless receiver laptops, tablet computers and mobile phones. Currently, there are various satisfy different functional requirements of the WiFi chip module on the market. After research and test the various WiFi transparent transmission modules, we finally adopted WG1300-UART-S0 to achieve seamless, transparent transmission between the serial port and WiFi.

The PLC Technology and Carrier Chip HT8550. Power line carrier [10] refers to the carrier analog or digital signal high-speed transmission technology in the existing power line, its greatest advantage is need not to re-build a special signal transmission line like other forms of communication. In principle, it is a communication mode that can be carried out with wires as long as there is a wire. Therefore, in the field of smart home, especially in the transformation of original home, it has an unparalleled advantage to other communication methods, as long as the home equipment internally equipped with power line carrier communication module, there is no need to set up the rest of the signal channel and it can achieve transformation. However, the original design of power line is used for power transmission, compared with other specialized communication channel and the transmission medium, the channel environment is more complex and the noise is more serious. Therefore, a reliable and real-time carrier through hearing mechanism must be established when building a communication network with the PLC technology. Numerous of documents and test shows that, in the power line carrier communication, signal-to-noise ratio(SNR) has great influence to the transmission distance and transmission reliability factors, and the key to improve the SNR lies in the low voltage power line carrier chip.

HT8550 is a relatively good chip, it is suitable for the complex situation of domestic power grid PLC data communication, and the analog circuits, digital signal processors and 8051MCU are fully implemented on a single chip. HT8550 uses orthogonal frequency division multiplexing (OFDM) modulation and demodulation, highly efficient and reliable forward error correction technology and flexible allocation of transmission mode, which can implement adaptive and reliable communication under the environment of serious signal attenuation and pulse jamming. In addition, compared to other electric power carrier communication chip, HT8550 has a big advantage, most of the traditional power carrier communication chip can achieve signal transmission, without signal processing functions, and HT8550 internally integrated the 8051 core, so it can implement most of the functions and applications on MAC layer and above protocol layer independently.

Networking Mode Based on WiFi and PLC. As the router and WiFi are widely existed in the family and company, we proposed a new smart home networking mode, namely PLC and WiFi combination of networking mode, design the access point module as a bridge to achieve the wireless network and power line. The whole communication system is shown in Fig. 1; access point receives commands from the control terminal (such as mobile phones, tablet PCs and PC) and sends them to the designated PLC terminal. PLC terminal connects to the equipment, receive commands from the control terminal to control the device, while checking the status of the device and feed it back to the control terminal through the access point.

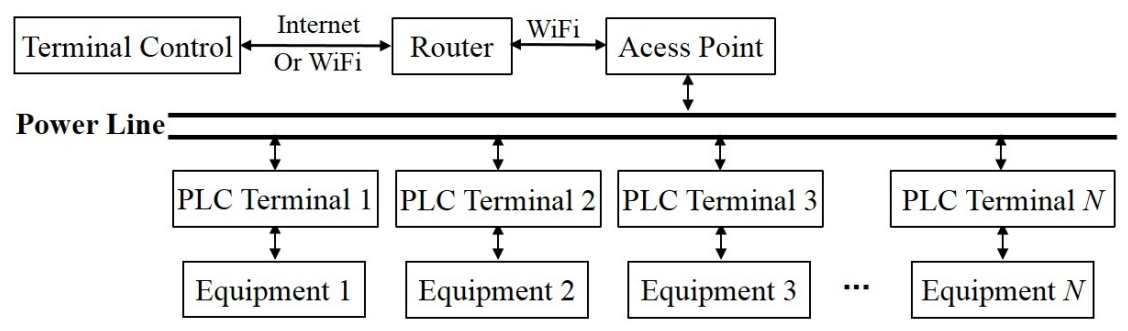

Fig. 1 Smart Home Network Architecture 


\section{The Design of the Key Hardware System}

The Design of Low Voltage Power Carrier Communication Module. PLC module structure is shown in Fig. 2.

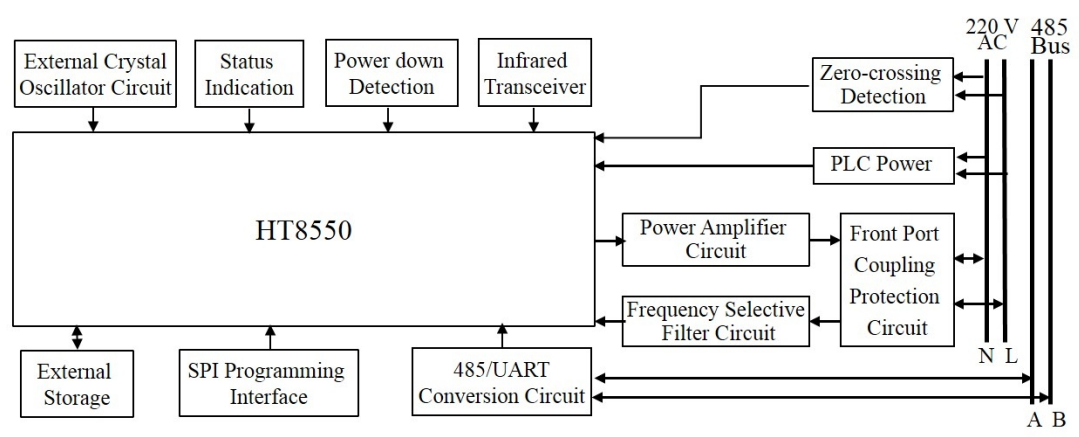

Fig. 2 PLC Module Structure

The main function of the low voltage power carrier communication module is to realize the modulation and demodulate signal, it can extend digital signal to spectrum carrier signal and transmitted it into $220 \mathrm{~V}$ AC power line, can also demodulate and process the power line carrier signal received from the $220 \mathrm{~V} \mathrm{AC}$, it is equipped with the circuit to implement electrical isolation, zero detection, signal amplification, signal indication and other auxiliary function. PLC module is mainly component with HT8550 carrier chip and drive circuit, power supply circuit, band-pass filter circuit, signal coupling circuit, zero crossing detection circuit, external crystal oscillator circuit, infrared transceiver circuit, 485/UART conversion circuit, power amplification circuit, EEROM external storage circuit and lights.

The Design of Access Point Module. Access point module is the central part of the system, and its main structure is shown in Fig. 3. It is mainly component of the WiFi chip, PLC chip and related peripheral circuit and can convert the WiFi signal to power line carrier signal to control the household appliances, perfectly implemented the interconnection between the power line carrier communication and Internet, solve the problem of heterogeneous network cannot control indoor power line communication. It allows users to transfer the control signal to the power line through the terminal client easily, and then control the smart home equipment.

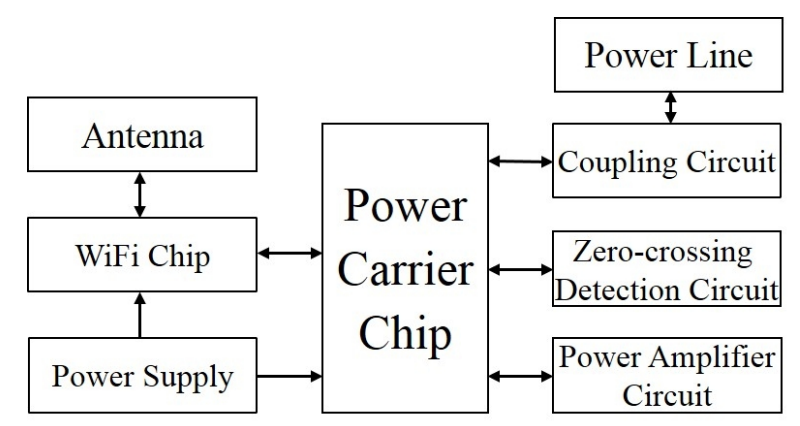

Fig. 3 Access Point Module

\section{Design of System Software}

Design of Lower Machine Software. In the program flow of machine software, after the system is powered, the application layer code will first perform the EUI address allocation and acquisition, after that, the application layer state to the idle state. When the control end request to send data to the power line, application layer performs the steps of: control end distribute the command parsing, application layer data framing, send data to PHY layer or net layer, setting the timeout, wait until the data is successfully transmitted, if successful or timeout, with layer back to the idle state. Application layer from the power line receiving data flow: query the event given by PHY layer or net layer; 
receive data of PHY layer or net layer, data analysis of application layer, if next operation is reported data, then reported data to the control terminal, application layer state back to idle state. The examples of data format: to control the light switch, for example, data transmission formats: control code for the reunification of the $0 \mathrm{x} 01$, length of the data is 1 . From left to right, the first representative control lamp is open or closed, represents 0,1 on behalf of; serial number of the 2-8 bits represent the lamp, for example: 1, 000, 000 said no. 1; 00000102 lights; 0011000 said six lights, and so on.

For example as follows:

1 control lights, sending [MAC] 0x680x01 0x01 command: 0x68 0x81 CS 0x16;

3 control lights off, send the command: 0x68 [MAC] 0x680x01 0x01 0x03 CS 0x16.

Design of Control Terminal Software. Control terminal software is a tool to realize intelligent control, in the whole system of the upper level is the direct interaction with the user equipment, so the friendly user interface and convenient intuitive operation experience is the first rule of the software design [11]. According to the smart home functional requirements [12], user experience, and other habits analysis, design the terminal control software based on the Android, the main structure of the software divided into account management, equipment management module and the access point module management. The design of the terminal control software system structure is shown in Fig. 4 .

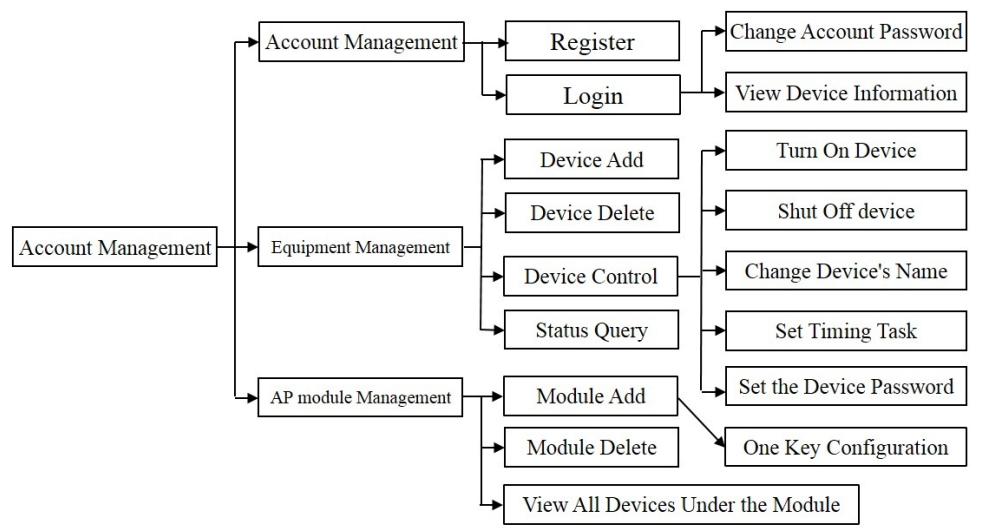

Fig. 4 Control Terminal Software Process

\section{Experimental Results Analysis}

Test records in real-time and reliability of the communication system are shown in Table 1.

Table 1 Test Records

\begin{tabular}{cccc}
\hline $\begin{array}{c}\text { Power line } \\
\text { length }(\mathrm{m})\end{array}$ & $\begin{array}{c}\text { The response time } \\
\text { is less than 1s }\end{array}$ & $\begin{array}{c}\text { Response time greater than 1s } \\
\text { or unable to respond }\end{array}$ & Qualified rate (\%) \\
\hline 5 & 96 & 4 & 96 \\
10 & 97 & 3 & 97 \\
20 & 94 & 6 & 94 \\
50 & 95 & 5 & 95 \\
100 & 94 & 6 & 94 \\
\hline
\end{tabular}

The data in Table 1 is the $5 \mathrm{~m}, 10 \mathrm{~m}, 20 \mathrm{~m}, 50 \mathrm{~m}$ and $100 \mathrm{~m}$ is get from the light equipped with PLC module for the 100 switch test, and we monitor the response time of the lights through the serial port. By comparing the response time of the different distances, we concluded that the system can meet the communication requirements of the smart home system and the places with high real-time requirements.

\section{Conclusions}

If uses WiFi or PLC technology into smart home system separately, its inherent shortcomings is can not meet the requirements of the smart home system, combine the two technology into a new smart home network mode and the system can realize the complementary advantages, we can use the 
widely, low cost and networking flexible characteristics of $\mathrm{WiFi}$, and also make full use the characteristic of PLC without re-wiring, little transformation. It is not only applies to the new home construction, but also applies to the existing residential renovation, fully embodies the smart home concept of energy-saving emission reduction and the purpose of people-oriented. Therefore, the smart home control system based on $\mathrm{WiFi}$ and PLC has good research value and market prospect.

\section{Acknowledgements}

This work was financially supported by higher vocational education brand professional construction project of Guangdong province (No. [2015]189).

\section{References}

[1] Shen Bin, Zhang Gui-qing, Wang Ming, et al. Design and Implementation of Smart Home Based on Internet of Things. Automation \& Instrumentation, 2013, (2): 6-10.

[2] Tong Xiao-yu, Fang Bing-yi, Zhang Yun-yong. Development analysis of internet of things smart home. Mobile Communications, 2010, (9): 16-20.

[3] Pan Zhao, Luo Xiao-qiao, Huang Jia, et al. Design of smart home controlling system based on GSM SMS. Electrion Measurement Technology, 2013, 36(6): 121-124.

[4] Yan Bing-yang. Design and implementation of remote meter reading security system based on $433 \mathrm{MHz}$ module. Ji'nan: Shandong Normal University, 2014.

[5] Qin Shi-huan, WU Guang-min. Design and Research of Smart Home System. Microprocessors, 2012, (2): 35-38.

[6] Hao Jie, Li Chao. The Application of Electric-network Carrier Technology in Smart Home. Journal of Zhejiang Wanli University, 2007, (3): 71-74.

[7] Zhou Xukun. Yang Guanlu. Research of smart home system based on ZigBee and PLC technology. Network and Communication, 2013, 32(9): 38-41.

[8] Zhou Xiang. Design and implementation of intelligent home control system based on WiFi-PLC. Yangzhou: Yangzhou University, 2010.

[9] Han Ting, Han Bing-jun, Zhang Ling. Coexistence Study for WiFi and ZigBee under Smart Home Scenarios. Network Infrastructure and Digital Content (IC-NIDC), 2012 3rd IEEE International Conference, 2012: 669-674.

[10] Han Jin-soo, Choi Chang-sic, Park Wan-ki. Smart Home Energy Management System Including Renewable Energy Based ZigBee and PLC. Consumer Electronics, IEEE Transaction, 2014, 62(2): 198-202.

[11] Huang Yu. A Thesis Submitted in Partial Fulfillment of the Requirements for the Degree of Master of Engineering. Wu Han: Huazhong University of Science and Technology, 2013.

[12] Wu Bi-zhao. The System Design and Implementation of Smart Home Control Terminal Based On Linux. Xi'an: University of Electronic Science and Technology of China, 2013. 